\title{
Pour une transition radicale et juste
}

For a Radical and Fair Transition

Association Science Technologie Société, Développement Durable et Territoires, Dossiers et Débats pour un Développement Durable, NaturesSciences-Sociétés-Dialogues, Vivagora, Coordination Eau Ile-de-France et L'eau est le pont

\section{(2) OpenEdition} Journals

\section{Édition électronique}

URL : http://journals.openedition.org/developpementdurable/9361

DOI : 10.4000/developpementdurable.9361

ISSN : 1772-9971

\section{Éditeur}

Association DD\&T

\section{Référence électronique}

Association Science Technologie Société, Développement Durable et Territoires, Dossiers et Débats pour un Développement Durable, Natures-Sciences-Sociétés-Dialogues, Vivagora, Coordination Eau lle-de-France et L'eau est le pont, «Pour une transition radicale et juste », Développement durable et territoires [En ligne], Vol. 3, n² 2 I Juillet 2012, mis en ligne le 04 janvier 2013, consulté le 04 mai 2019 URL : http://journals.openedition.org/developpementdurable/9361 ; DOI : 10.4000/ developpementdurable.9361

Ce document a été généré automatiquement le 4 mai 2019.

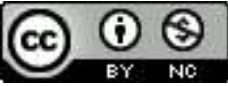

Développement Durable et Territoires est mis à disposition selon les termes de la licence Creative Commons Attribution - Pas d'Utilisation Commerciale 4.0 International. 


\title{
Pour une transition radicale et juste
}

\author{
For a Radical and Fair Transition
}

\begin{abstract}
Association Science Technologie Société, Développement Durable et Territoires, Dossiers et Débats pour un Développement Durable, NaturesSciences-Sociétés-Dialogues, Vivagora, Coordination Eau Ile-de-France et L'eau est le pont
\end{abstract}

\section{NOTE DE L'ÉDITEUR}

Cette déclaration fait suite au Forum des associations « Repenser le développement : la société civile s'engage » qui s'est tenu en janvier 2011 à la Cité internationale universitaire, à Paris (cf. http://www.nss-dialogues.fr/Presentation,1). Cette déclaration est destinée à être signée par toute association qui souhaite s'engager dans la démarche décrite ci-dessus. Pour signer la déclaration : http://www.nss-dialogues.fr/DeclarationPour-une-transition

1 Il existe en France un mouvement associatif fort de centaines d'associations qui anime la réflexion sur le mode de développement de la société française actuelle et les problèmes qu'il soulève tant en interne que dans les rapports de la France avec tous les pays du monde, et qui agit en vue de sa transformation. Au-delà de leur extrême diversité, les associations qui en constituent la base active partagent les mêmes buts et se reconnaissent dans un certain nombre de valeurs communes. Elles estiment que la crise multiforme que nous vivons exige une analyse critique radicale $\mathrm{du}$ mode de développement et du type de société qui y ont conduit, et une transition tout aussi radicale vers un mode de développement et un type de société qui répondent pleinement aux questions soulevées par les dégâts écologiques, économiques et sociaux qu'ils ont produits. Pour elles, la question sociale, la question du travail, la question de la démocratie et la question environnementale forment un tout et doivent être considérées et traitées ensemble car elles procèdent de la même cause, l'exaspération de la poursuite du profit à court terme. 
Elles sont tout particulièrement conscientes des inégalités sociales qui sont à la fois à la base et la conséquence de ces dégâts, conscientes du risque que les indispensables transformations à venir des modes de production et de consommation les aggravent et donc tout particulièrement soucieuses que soient réalisées les conditions d'une transition juste. Cela veut dire que la transition à faire doit être impérativement placée sous le signe de la solidarité et c'est pourquoi elles mettent la solidarité au cœur de leur action. Cette exigence les conduit à accorder une attention prioritaire au politique, car une transition juste suppose une action forte des pouvoirs publics, à tous leurs niveaux, Europe comprise.

3 Vivement attachées à la démocratie, elles sont préoccupées par les risques de dérives autoritaires, oligarchiques ou populistes que lui font courir les tensions produites par les conséquences de la crise et les choix à faire pour en sortir par le haut. Elles en appellent à une mobilisation citoyenne.

$4 \quad$ Y travaillant, l'organisant, en connaissant les difficultés et les ressorts, elles estiment avoir un rôle privilégié à jouer dans les processus démocratiques lorsqu'ils concernent des domaines en rapport avec la question du développement et avec les questions de tous ordres (économiques, politiques, sociales, culturelles) en découlant. A ce titre, elles revendiquent l'instauration, dans le cadre de la démocratie représentative, d'une démocratie participative dans laquelle elles aient pleinement leur place.

5 Mais leur participation doit être bien prise pour ce qu'elle doit être: les expériences qu'elles tirent de leur engagement sociétal leur donnant une capacité d'analyse et d'expertise propre, citoyenne, c'est bien d'un espace d'expression d'une citoyenneté prenant en main la transition vers une démocratie écologique et sociale qu'elles se réclament. Cette intervention citoyenne revêt une importance toute particulière à propos de la question de la recherche et de l'innovation, car elle contient ni plus ni moins celle du choix de société.

6 Le mouvement associatif qui veut « repenser le développement » agit comme un levain; il contribue à esquisser le tableau d'un souhaitable possible; il représente une masse d'énergie sociale considérable; il touche toutes les facettes de la vie en société et de la vie personnelle, les irrigue et s'en nourrit; il met en action des valeurs essentielles pour la vie en commun; il mobilise des sommes de connaissances et de compétences, des ressources psychiques, émotionnelles, affectives multiples et fortes; il crée du lien social. Tout cela, avec l'efficacité de son omniprésence, souvent discrète, et de sa diversité adaptative.

7 L'importance des enjeux, l'urgence des décisions à prendre, l'énormité des obstacles à surmonter lui font aujourd'hui obligation d'unifier ses forces, d'être une caisse de résonnance pour toutes les initiatives qui contribuent d'ores et déjà à donner corps à la transition souhaitable, de se donner plus de visibilité et, par là, de contribuer à renforcer la place de la société civile dans le débat démocratique.

8 En conséquence, les associations signataires déclarent adhérer à une démarche visant à promouvoir une transition écologique et sociale radicale, juste et démocratique et s'engagent à coopérer activement entre elles pour faire en sorte que leurs réflexions et leurs actions respectives y contribuent. 


\section{AUTEURS}

\section{ASSOCIATION SCIENCE TECHNOLOGIE SOCIÉTÉ}

L'Association Science Technologie Société (ASTS) est une association à but non lucratif régie par la loi de 1901, intervenant dans le champ de la médiation scientifique et du débat citoyen. Association de Jeunesse et d'éducation Populaire, elle intervient dans toute la France. http:// www.asts.asso.fr

\section{DÉVELOPPEMENT DURABLE ET TERRITOIRES}

L'Association « Développement durable et territoires » (DD\&T) réunit une quarantaine de chercheurs en sciences humaines et sociales, issus de différentes disciplines, et principalement des Universités et laboratoires de recherches de la région Nord-Pas-de-Calais. Elle édite la revue Développement durable et territoires (http://developpementdurable.revues.org), organise des journées d'études (http://www.meshs.fr/page.php?r=24\&id=1129\&lang=fr) et participe à différents projets de recherche.

\section{DOSSIERS ET DÉBATS POUR UN DÉVELOPPEMENT DURABLE}

L'Association 4D a été créée en 1993, après le sommet de Rio, afin de constituer un réseau citoyen pour la promotion du développement durable et le suivi des engagements pris par la France, comme par les autres états membres de l'ONU. http://www.association4d.org/

\section{NATURES-SCIENCES-SOCIÉTÉS-DIALOGUES}

Créée en 1991, l'association Natures Sciences Sociétés - Dialogues est ouverte aux chercheurs, aux enseignants, aux praticiens. Elle veut offrir un lieu de rencontres, de dialogues et de confrontations : (1) pour construire l'interdisciplinarité que requièrent les multiples questions posées par tout ce qui a trait aux rapports entre l'homme et son environnement ; (2) pour confronter les points de vue des praticiens et des scientifiques ; (3) pour forger les bases culturelles d'un nouveau regard de l'homme sur la nature et sur lui-même. Pour cela, elle prend toute initiative destinée à développer ce dialogue ; elle soutient des séminaires, des colloques, une revue interdisciplinaire (Natures Sciences Sociétés) et une collection d'ouvrages (« Indisciplines »). http://www.nss-dialogues.fr/

\section{VIVAGORA}

Créée en 2003, l'association Vivagora agit pour mettre en démocratie les choix scientifiques et techniques. Outre son soutien à la veille sur les technologies émergentes et leur gouvernance, à l'ouverture de l'expertise sur les dimensions sociales, sanitaires et écologiques, VivAgora développe des méthodes de dialogues entre les scientifiques, industriels et les citoyens pour ajuster l'innovation aux territoires et à leurs cultures propres. http://www.vivagora.fr/

\section{COORDINATION EAU ILE-DE-FRANCE}

Crée en 2008, l'association Coordination eau Ile-de-France vise à mettre en réseau les associations d'usagers citoyens de l'eau, quelle que soit leur approche (socio-économique, environnementale, consumériste, culturelle...), à rendre visible ce mouvement et à en faire un acteur incontournable 
de toutes les décisions concernant l'eau et l'assainissement en Ile-de-France. http:// eauidf.blogspot.fr/

\section{L'EAU EST LE PONT}

«L'eau est le pont » est un projet associatif pour "réveiller" dans l'esprit de chacun la conscience de l'eau, le sentiment de sa valeur, la réalité de sa place dans notre environnement, sur la planète, dans notre vie, dans notre imaginaire... http://leauestlepont.free.fr/ 